\title{
Serological Evidence of Coxiella burnetii and SARS- CoV-2 Co-infection: A Case Report
}

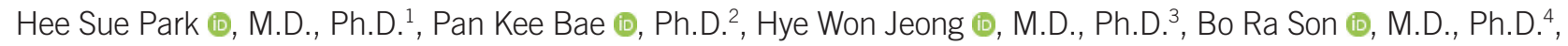 \\ and Kyeong Seob Shin ${ }^{\oplus}$, M.D., Ph.D. ${ }^{4}$ \\ ${ }^{1}$ Department of Laboratory Medicine, Chungbuk National University Hospital, Cheongju, Korea; ${ }^{2}$ BioNano Health Guard Research Center (H-GUARD), \\ Daejeon, Korea; ${ }^{3}$ Department of Internal Medicine, Chungbuk National University College of Medicine, Cheongju, Korea; ${ }^{4}$ Department of Laboratory \\ Medicine, Chungbuk National University College of Medicine, Cheongju, Korea
}

\section{Dear Editor,}

The most common symptoms of coronavirus disease 2019 (COVID-19) are respiratory symptoms that are not easily distinguishable from those of other acute respiratory infections [1]. As bacterial/fungal co-infections are reported in $8 \%$ of COVID-19 patients, diagnosing them is critical for appropriate treatment [2]. Pathogens that cause co-infection with severe acute respiratory syndrome coronavirus 2 (SARS-CoV-2) include influenza A/B viruses, Mycoplasma pneumoniae, Acinetobacter baumannii, Candida albicans, and Legionella pneumophila [2]. Q fever is asymptomatic in approximately $60 \%$ cases, but a flu-like illness with high fever, myalgia, headache, and cough, which lasts for one to three weeks, may occur in acute infection and then resolve spontaneously [3]. We report the case of a 37-year-old man who was diagnosed as having Coxiella burnetii and SARSCoV-2 co-infection. To the best of our knowledge, this is the first such report in Korea. The study was approved by the Institutional Review Board of the Chungbuk National University Hospital, Cheongju, Korea (IRB number: 2020-03-025).

A 37-year-old man presented to the Chungbuk National University Hospital in May 2020 with fever, cough, and sputum development, which had started three days before hospital presentation. The patient was a farmer, with no epidemiologic link to COVID-19-confirmed cases. Physical examination revealed a temperature of $38.8^{\circ} \mathrm{C}$, blood pressure of $126 / 64 \mathrm{~mm} \mathrm{Hg}$, pulse rate of $95 /$ minute, and respiratory rate of 18 /minute. A complete blood count revealed a hemoglobin level of $151 \mathrm{~g} / \mathrm{L}$, white blood cell count of $2.32 \times 10^{9}$ cells $/ \mathrm{L}$ (absolute neutrophil count $1.37 \times$ $10^{9}$ cells/L, absolute lymphocyte count $0.64 \times 10^{9} \mathrm{cell} / \mathrm{L} / \mathrm{L}$ ), and platelet count of $116 \times 10^{9} / \mathrm{L}$. Other blood tests revealed elevated levels of C-reactive protein $(50.5 \mathrm{mg} / \mathrm{L})$ and lactate dehydrogenase (12.07 $\mu \mathrm{kat} / \mathrm{L})$ and slightly elevated levels of D-dimer (12.05 $\mathrm{nmol} / \mathrm{L})$, AST (1.12 $\mu \mathrm{kat} / \mathrm{L})$, and ALT (0.83 $\mu \mathrm{kat} / \mathrm{L})$. The patient's prothrombin time and activated partial-thromboplastin time tests were within the reference ranges (Table 1). Chest X-ray showed no active lung lesion. A real-time reverse transcription (real-time RT-PCR) (Allplex 2019-nCoV Assay, Seegen, Seoul, Korea) test for SARS CoV 2 was performed on admission day. The result was negative in a naso/oropharyngeal swab and positive in sputum. Multiplex RT-PCR results for M. pneumoniae, Chlamydia pneumoniae, L. pneumophila, Bordetella pertussis, Streptococcus pneumoniae, Haemophilus influenzae (Allplex PneumoBacter Assay, Seegen) and influenza A/B virus (Sofia fluorescence immunoassay, Quidel, San Diego, CA, USA) were all negative. Considering the patient's occupation, serological tests for C. burnetii, Leptospira interrogans, and Orientia tsutsugamushi
Received: September 23, 2020

Revision received: November 18, 2020

Accepted: March 15, 2021

Corresponding author: Kyeong Seob Shin, M.D., Ph.D.

Department of Laboratory Medicine, Chungbuk National University College of Medicine, 1 Chungdae-ro, Seowon-gu, Cheongju 28644, Korea

Tel: +81-43-269-6240

Fax: +81-43-271-5243

E-mail: ksshin@chungbuk.ac.kr 


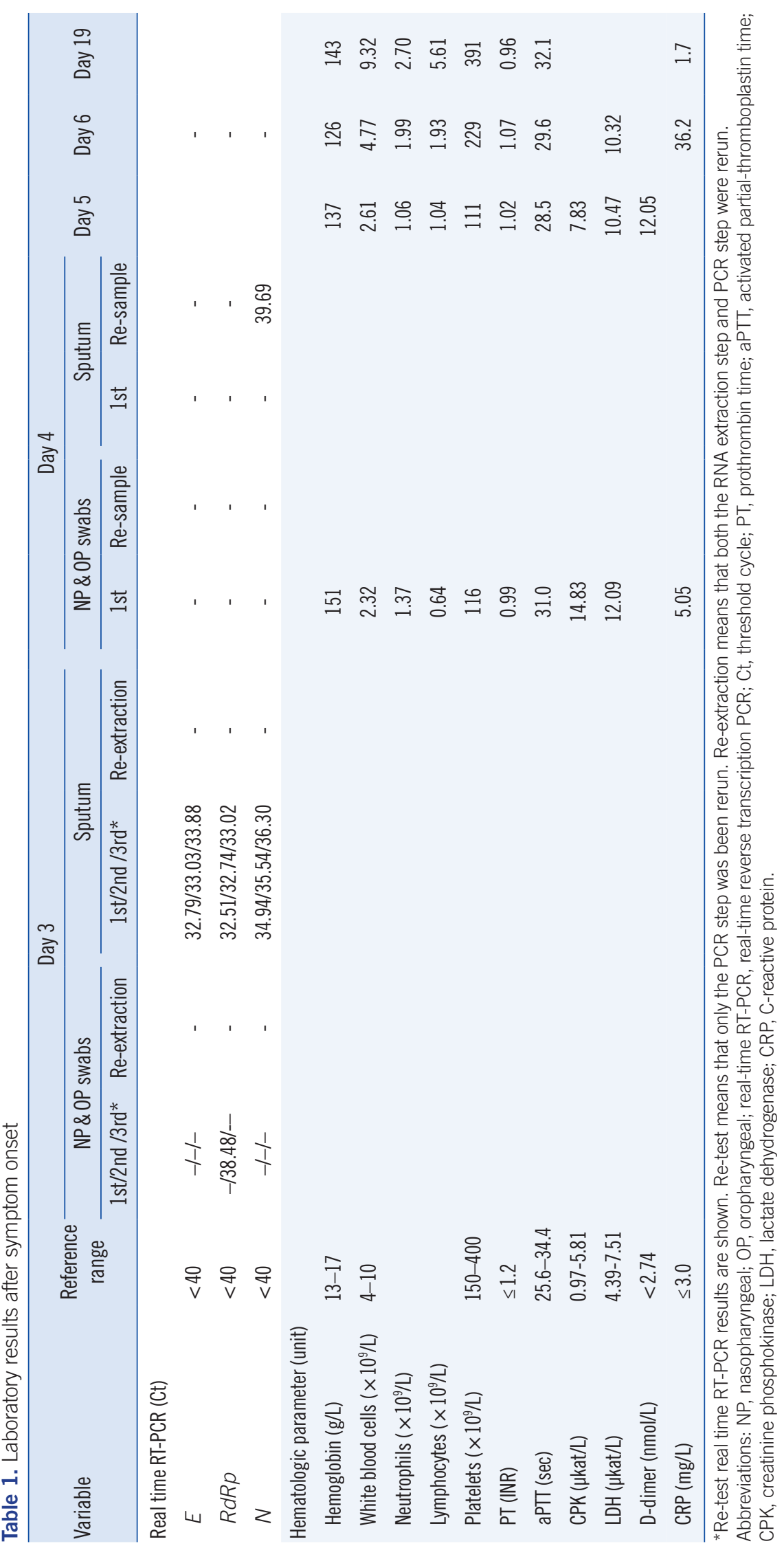


were performed on admission day and were all negative.

The next day, SARS-CoV-2 RT-PCR test results were negative. To rule out a false-positive result in the first RT-PCR test, we reextracted and re-tested the original samples. After re-extraction, the sputum tested negative. In addition, serological antibody tests for SARS-CoV-2 infection were performed to rule out a false-positive RT-PCR result. A sandwich ELISA targeting the SARS-CoV-2 receptor binding domain (RBD) of the spike protein (SP) was conducted the next day. Briefly, the SARS-CoV-2 RBD antigen was attached to a 96-well plate and diluted serum was applied. After washing to remove unbound substance, the detection antibody (horseradish peroxidase-conjugated anti-human IgG or lgM) was added. After washing away excess detection antibody, the optical density at $450 \mathrm{~nm}$ in each well was measured using a microplate reader. The anti-RBD IgG antibody test was positive on the day after admission (Fig. 1). Based on the positive SARS-CoV-2 RT-PCR result in sputum and positive sandwich ELISA result, the patient was diagnosed as having SARS-CoV-2 infection. Follow-up serological tests showed patient seroconversion indicating $C$. burnetii infection. Therefore, co-infection with $C$. burnetii and SARS-CoV-2 was confirmed in the follow-up period.

Although the severity of COVID-19 varies from mild to lifethreatening, bacterial or fungal co-infection in COVID-19 patients increases the risk of mortality [4]. Therefore, clinicians should consider the variable clinical severity of COVID-19 and the possibility of co-infection, which may cause the same symptoms as COVID-19 but can aggravate the patient's condition and require additional laboratory testing for diagnosis.

Real-Time RT-PCR is a standard method for diagnosing
SARS-CoV-2 infection, as it gives minimal false-positive results [5]. Considering that negative conversion of real-time RT-PCR test results takes more than two weeks for SARS-CoV-2 infection [6], the patient might have had SARS-CoV-2 infection in the past. On days 15 to 29 of COVID-19, the sensitivity of real-time RT-PCR is $70.7 \%$, whereas that of ELISA is $100 \%$ [7]. In serological tests for SARS-CoV-2, various target proteins, such as RBD, nucleocapsid protein, and SP, can be used, and, when these tests are used in combination with molecular tests, the sensitivity and specificity of COVID-19 diagnosis are increased [7]. In our case, a false-positive real-time RT-PCR result could not be ruled out, but SARS-CoV-2 infection was assumed, considering the results of additional serological tests. We believe that the negative conversion of the real-time RT-PCR result was due to a low viral load or virus remnant. Anti-SARS-CoV-2 IgM is less sensitive than IgG [8], and a negative IgM result on days 3 and 4 is considered false. It is necessary to further evaluate the diagnostic performance of serological tests for COVID-19.

In $Q$ fever, serological tests have been used to diagnose acute infection, and seroconversion from negative to positive occurs one to three weeks after symptom onset [3]. Although viral loads do not differ between asymptomatic and symptomatic COVID-19 patients, our patient's symptoms at the time of hospital presentation are more likely to have been due to $Q$ fever [9]. Other laboratory results were nonspecific, but lymphopenia was notable on admission. Lymphopenia is rarely observed in $Q$ fever but is common in COVID-19, for which it is a prognostic indicator [10].

To the best of our knowledge, this is the first report on $C$. burnetii and SARS-CoV-2 co-infection, and serologic testing played

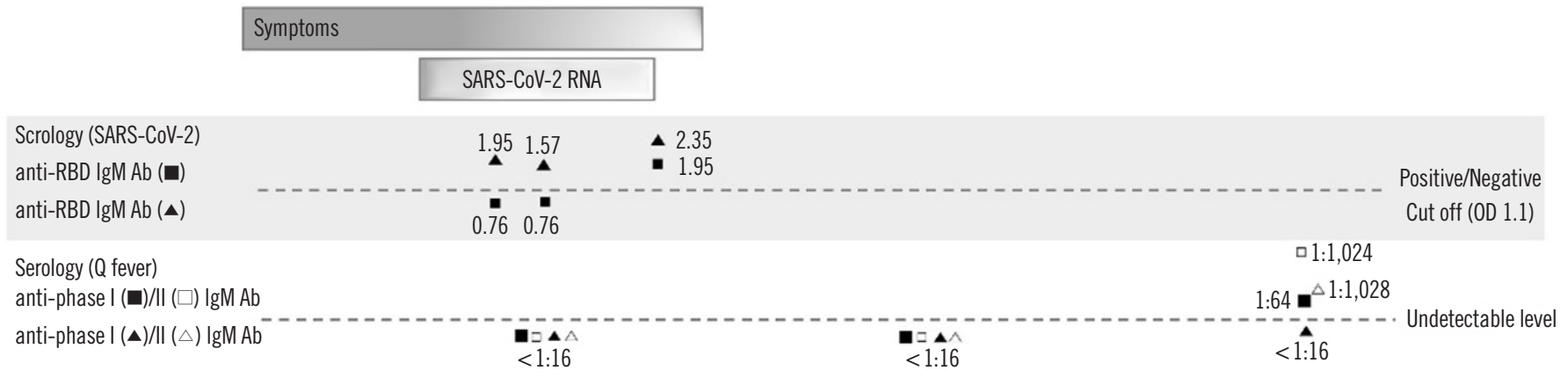

\begin{tabular}{|c|c|c|c|c|c|c|c|c|c|c|c|c|c|c|c|c|c|c|c|c|}
\hline 0 & 1 & 2 & 3 & 4 & 5 & 6 & 7 & 8 & 9 & 10 & 11 & 12 & 13 & 14 & 15 & 16 & 17 & 18 & 19 & 20 \\
\hline
\end{tabular}

Fig. 1. Serological diagnosis of co-infection with Coxiella burnetii and SARS-CoV-2. Serological tests for SARS-CoV-2 IgM/lgG anti-RBD and C. burnetii were positive, indicating co-infection. Abbreviations: SARS-CoV-2, severe acute respiratory syndrome coronavirus 2; RBD, SARS-CoV-2 receptor binding domain. 
an important role in the diagnosis. For accurate COVID-19 diagnosis, clinicians should consider a multidisciplinary approach and utilize accurate and rapid diagnostic tools.

\section{ACKNOWLEDGEMENTS}

We thank all clinicians and laboratory technologists in Chungbuk National University Hospital and BioNano Health Guard Research Center.

\section{AUTHOR CONTRIBUTIONS}

Park HS designed the study and wrote the manuscript; Bae PK carried out the experiment and analyzed the data; Son BR collected the data; Jeong HW provided clinical information and discussed the manuscript; Shin KS designed the study and edited the manuscript. All authors have read the approved the final manuscript.

\section{CONFLICTS OF INTEREST}

The authors declare no conflict of interest.

\section{RESEARCH FUNDING}

Not applicable.

\section{ORCID}

Hee Sue Park

https://orcid.org/0000-0002-8378-6066
Pan Kee Bae

https://orcid.org/0000-0003-4488-5261

Hye Won Jeong

Bo Ra Son

Kyeong Seob Shin https://orcid.org/0000-0002-1063-8476

https://orcid.org/0000-0001-9020-4303

https://orcid.org/0000-0002-1680-1510

\section{REFERENCES}

1. Zha L, Shen J, Tefsen B, Wang Y, Lu W, Xu Q. Clinical features and outcomes of adult COVID-19 patients co-infected with Mycoplasma pneumoniae. J Infect 2020;81:e12-5.

2. Rawson TM, Moore LS, Zhu N, Ranganathan N, Skolimowska K, Gilchrist $\mathrm{M}$, et al. Bacterial and fungal co-infection in individuals with coronavirus: a rapid review to support COVID-19 antimicrobial prescribing. Clin Infect Dis 2020;71:2459-68.

3. Gikas A, Kokkini S, Tsioutis C. Q fever: clinical manifestations and treatment. Expert Rev Anti Infect Ther 2010;8:529-39.

4. Zhou F, Yu T, Du R, Fan G, Liu Y, Liu Z, et al. Clinical course and risk factors for mortality of adult inpatients with COVID-19 in Wuhan, China: a retrospective cohort study. Lancet 2020;395:1054-62.

5. Hong KH, Lee SW, Kim TS, Huh HJ, Lee J, Kim SY et al. Guidelines for laboratory diagnosis of Coronavirus Disease 2019 (COVID-19) in Korea. Ann Lab Med 2020;40:351-60

6. Carmo A, Pereira-Vaz J, Mota V, Mendes A, Morais C, da Silva AC, et al. Clearance and persistence of SARS-CoV-2 RNA in patients with COVID-19. J Med Virol 2020;92: 2227-31.

7. Lou B, Li T, Zheng S, Su Y, Li Z, Liu W, et al. Serology characteristics of SARS-CoV-2 infection since exposure and post symptom onset. Eur Respir J 2020;56:2000763.

8. Zhang Z, Hou Y, Li D, Li F. Diagnostic efficacy of anti-SARS-CoV-2 IgG/ IgM test for COVID-19: a meta-analysis. J Med Virol 2020; 93:366-74

9. Zou L, Ruan F, Huang M, Liang L, Huang H, Hong Z, et al. SARS-CoV-2 viral load in upper respiratory specimens of infected patients. N Engl J Med 2020;382:1177-9.

10. Terpos E, Ntanasis-Stathopoulos I, Elalamy I, Kastritis E, Sergentanis TN, Politou M, et al. Hematological findings and complications of COVID-19. Am J Hematol 2020; 95:834-47. 